\title{
Distributed Balanced Photodetectors for High-Performance RF Photonic Links
}

\author{
M. Saiful Islam, Student Member, IEEE, Tai Chau, Antonino Nespola, Sagi Mathai, Andrew R. Rollinger, \\ William R. Deal, Tatsuo Itoh, Fellow, IEEE, Ming C. Wu, Member, IEEE, \\ Deborah L. Sivco, and Alfred Y. Cho
}

\begin{abstract}
A novel velocity-matched distributed balanced photodetector with a 50- $\Omega$ coplanar waveguide output transmission line has been experimentally demonstrated in the InP-InGaAs material system. Distributed absorption and velocity matching are employed to achieve high-saturation photocurrent. A common mode rejection ratio of $27 \mathrm{~dB}$ has been achieved. The RF link experiment conducted at $6.48 \mathrm{GHz}$ shows that the laser intensity noise has been suppressed by more than $17 \mathrm{~dB}$.
\end{abstract}

Index Terms - Analog fiber-optic links, balanced photodetectors, noise suppression, optical receivers, RF photonics.

\section{INTRODUCTION}

B ALANCED PHOTODETECTORS are of great interest to analog fiber-optic links because they can suppress laser relative intensity noise (RIN) and amplified spontaneous emission noise (ASE) from erbium-doped fiber amplifiers (EDFA's) [1]. Because balanced photodetectors can achieve shot noise-limited link performance, we can continue to improve the noise figure and the spurious-free dynamic range (SFDR) of externally modulated links by increasing the power of the optical carrier. Therefore, balanced photodetectors with broad bandwidth and high-saturation photocurrents are particularly important for analog fiber-optic link applications. Though discrete balanced photodetectors with high-saturation power have been reported, their bandwidth is limited [2]. Most of the reported integrated balanced receivers suffer from low saturation power and are not suitable for analog links [3]-[5]. Previously, we have reported a velocity-matched distributed photodetector (VMDP) with a peak saturation photocurrent of $56 \mathrm{~mA}$ and a 3-dB bandwidth of $49 \mathrm{GHz}$ [6]. Recently, InP-based long wavelength VMDP has also been reported [7]. Compared with other photodetector structures, the VMDP is more suitable for implementing the balanced photodetection since it has separate optical and microwave waveguides. In this letter, we propose and demonstrate a novel, monolithic distributed balanced photodetector that can simultaneously

Manuscript received September 24, 1998; revised December 17, 1998. This work was supported by the Office of Naval Research MURI on RF Photonics, by the National Radio Astronomy Observatory (NRAO), by JESP, and by UC MICRO.

M. S. Islam, T. Chau, S. Mathai, A. R. Rollinger, W. R. Deal, T. Itoh, and M. C Wu are with the Department of Electrical Engineering, University of California at Los Angeles, Los Angeles, CA 90095-1594 USA.

A. Nespola is with the Politecnico di Torino, Dipartimento di Elettronica, I-100129 Torino, Italy.

D. L. Sivco and A. Y. Cho are with Lucent Technologies, Bell Laboratories, Murray Hill, NJ 07974 USA.

Publisher Item Identifier S 1041-1135(99)02508-2.

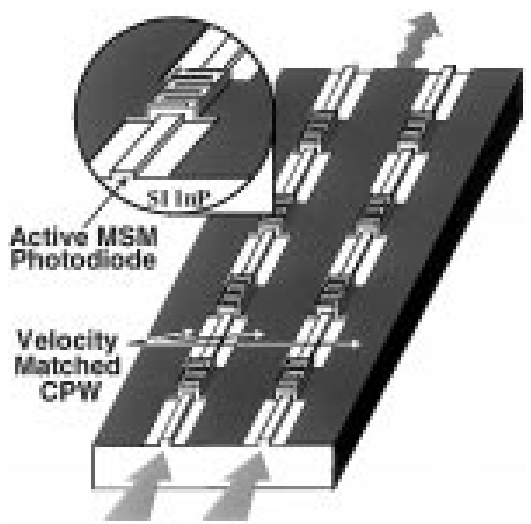

(a)

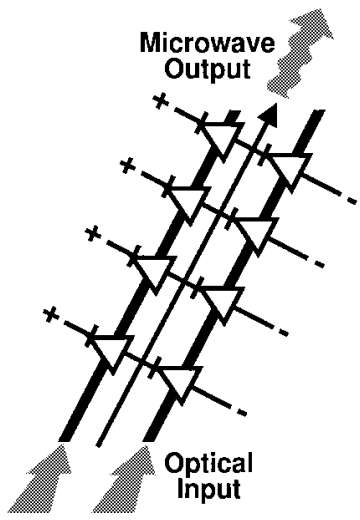

(b)

Fig. 1. (a) Schematic structure of the distributed balanced photodetector. Inset shows the active region with an MSM photodiode. (b) Photodiodes connected to the optical waveguide in balanced detection mode.

achieve high-saturation photocurrent and large bandwidth. A common mode rejection ratio of $27 \mathrm{~dB}$ and a noise suppression of $17 \mathrm{~dB}$ have been experimentally demonstrated.

\section{DESIGN AND FABRICATION}

Fig. 1 shows the schematic of the distributed balanced photodetector. It consists of two input optical waveguides, two arrays of high-speed metal-semiconductor-metal (MSM) photodiodes distributed along the optical waveguides, and a $50-\Omega$ coplanar waveguide (CPW) output transmission line. The diodes are $23 \mu \mathrm{m}$ long and $5 \mu \mathrm{m}$ wide. The MSM fingers with $1 \mu \mathrm{m}$ width and $1-\mu \mathrm{m}$ spacing are patterned by optical lithography. The central conductor of the CPW has a width of 55 
$\mu \mathrm{m}$ and the separation between the central conductor and the ground conductors is $85 \mu \mathrm{m}$. The $10.5-\mu \mathrm{m}$ overlap of the MSM fingers is designed to provide the required capacitive loading for velocity matching. Unlike previously reported slow-wave CPW [8] that ignored the resistance of the MSM photodiode fingers, finite metal thickness, and transmission line discontinuities in their quasistatic calculations, we used a full-wave analysis to achieve the velocity and impedance matching.

The distributed balanced photodetector inherits the basic advantages of the VMDP, namely, high-saturation photocurrent, high quantum efficiency, and large bandwidth. It should be noted that even though only the difference current (ac signal) is collected in the balanced photodetector, the dc light is still absorbed in the photodiodes. As a result, high-dc saturation photocurrent is required for the distributed balanced photodetectors. By coupling only a small fraction of light from the passive waveguide to each individual photodiode, the photodiodes are kept below saturation even under intense optical input. The bandwidth of the distributed balanced photodetector remains high because of the velocity matching.

Beam propagation method (BPM) simulation was used to investigate the optical properties of the balanced VMDP. The simulation shows that an input beam with TM polarization contributes to the optimum performance. Details of the simulation of the polarization dependence of absorption and optical coupling loss between photodiodes will be discussed elsewhere. The optical waveguide consists of the following: a 200 -nm-thick $\operatorname{In}_{0.52} \mathrm{Al}_{0.37} \mathrm{Ga}_{0.11}$ As lower cladding layer, a 500-nm-thick $\operatorname{In}_{0.52} \mathrm{Al}_{0.178} \mathrm{Ga}_{0.302} \mathrm{As}$ core region, a 200-nmthick $\mathrm{In}_{0.52} \mathrm{Al}_{0.37} \mathrm{Ga}_{0.11}$ As first upper cladding layer, and a thin $\operatorname{In}_{0.52} \mathrm{Al}_{0.48} \mathrm{As}$ second upper cladding layer. The $150-\mathrm{nm}$ thick absorption region is located on top of the waveguide for evanescent coupling. Since the Schottky barrier height of most metals on the InGaAs is typically between $0.2-0.3 \mathrm{eV}$, an $\mathrm{In}_{0.52} \mathrm{Al}_{0.48} \mathrm{As}$ cap layer is used to increase the Schottky barrier height and therefore decrease the dark current in the photodiodes [9]. A graded layer is incorporated in the structure to reduce the minority carrier trapping at the InAlAs-InGaAs band-edge discontinuity.

Fabrication of the receiver started with removing the InGaAs layer except in the active areas of the photodiodes. Ridge waveguides with 100-nm ridge height were formed by wet chemical etching. The active regions of the photodiodes were defined by opening $6 \times 23 \mu \mathrm{m}$ windows on the 150 -nm-thick silicon nitride $\left(\mathrm{Si}_{3} \mathrm{~N}_{4}\right)$ film deposited by plasma-enhanced chemical vapor deposition (PECVD). Buffered HF was used to remove the $\mathrm{Si}_{3} \mathrm{~N}_{4}$ in the windows. The Ti-Au electrodes and contact pads of the MSM photodiodes were then delineated by standard liftoff processes. The tips of the MSM fingers are placed on top of $\mathrm{Si}_{3} \mathrm{~N}_{4}$ to suppress the soft breakdown and enable the MSM diodes to operate over a wider range of bias voltage [10]. Finally, a 350-nm-thick coplanar waveguide was formed by standard lift-off process to connect the distributed balanced photodetectors.

\section{MEASUREMENTS AND DisCUSSIONS}

The balanced VMDP exhibits very good electrical and optical characteristics. The dark current is measured to be 28

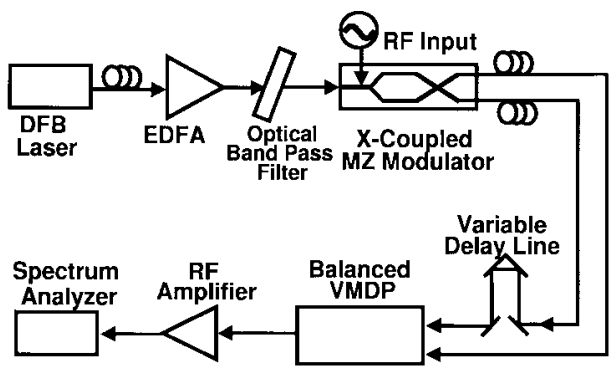

(a)

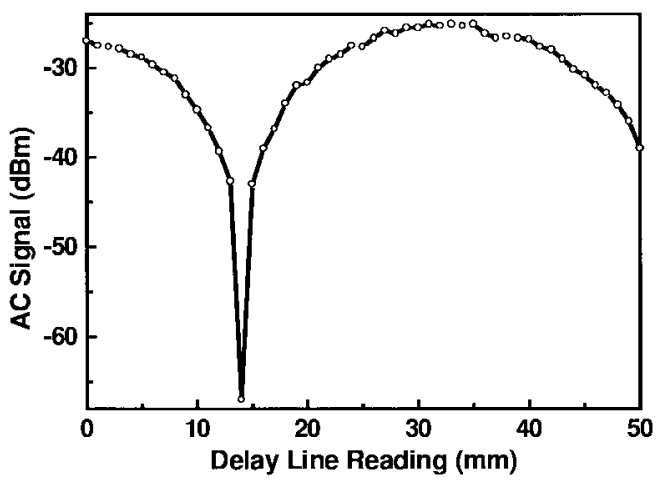

(b)

Fig. 2. (a) Balanced detection setup. The complimentary input signals are produced by the X-coupled MZ modulator. (b) Peak power of ac signal in balanced mode versus the delay. For RF signal at $8 \mathrm{GHz}$, the period was measured to be $38.75 \mathrm{~mm}$, which is very close to the theoretical value. The power difference between $0^{\circ}$ and $180^{\circ}$ phase difference is more than $44 \mathrm{~dB}$.

$\mu \mathrm{A} / \mathrm{cm}^{2}$ at $10-\mathrm{V}$ bias, the lowest reported for InAlAs-InGaAs MSM photodiodes. By coupling light directly from a lensed fiber, the average dc responsivity (15 devices tested) was measured to be $0.45 \mathrm{~A} / \mathrm{W}$ at $8-\mathrm{V}$ bias. Responsivity as high as $0.6 \mathrm{~A} / \mathrm{W}$ has been observed in some devices. The photo response of a laser beam with $\mathrm{TM}$ polarization is measured to be $\sim 1.7 \mathrm{~dB}$ higher than that of TE polarization. The coupling efficiency of the lensed fibers in our setup was calculated to be $\sim 50 \%$. With antireflection (AR) coating to the balanced receiver facet (30\% Fresnel loss), the average responsivity can be increased to $0.64 \mathrm{~A} / \mathrm{W}$.

An HP 8510C network analyzer was used to measure the characteristic impedance and the microwave return loss $\left(S_{11}\right)$ of the balanced receiver. The characteristic impedance of the receiver is very well matched to $50 \Omega$. The $S_{11}$ is as low as $-30 \mathrm{~dB}$ from $45 \mathrm{MHz}$ to $40 \mathrm{GHz}$. The $\mathrm{CPW}$ has very low insertion loss. The measured $\mathrm{S}_{12}$ shows a drop of only $0.6 \mathrm{~dB}$ from $45 \mathrm{MHz}$ to $40 \mathrm{GHz}$.

The frequency response of the balanced VMDP was first characterized with light coupled to one waveguide only. Using the optical heterodyne technique with two external cavity tunable semiconductor lasers at $1.55 \mu \mathrm{m}$, the $3-\mathrm{dB}$ bandwidth was found to be $16 \mathrm{GHz}$ for both photodetector arrays. The bandwidth is currently limited by the carrier transit time of the MSM photodiodes. Since our bandwidth of the capacitance loaded CPW is much greater than $40 \mathrm{GHz}$, the bandwidth of the balanced VMDP can be increased by scaling down the MSM photodiodes. Theoretical simulation indicates that bandwidth $>100 \mathrm{GHz}$ is achievable. 


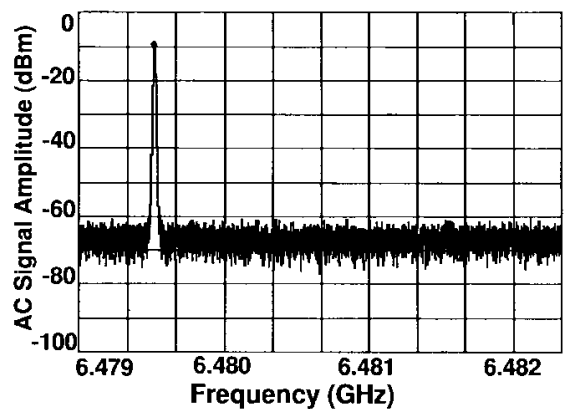

(a)

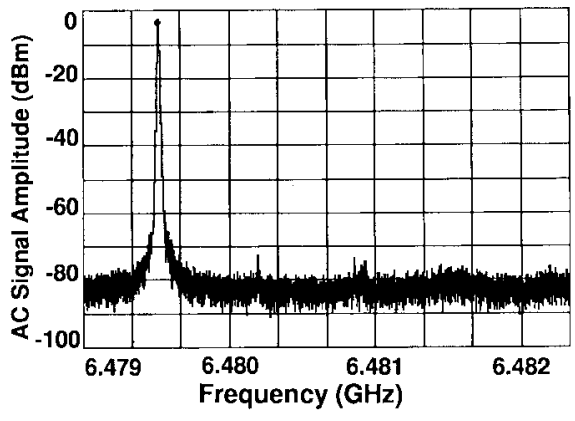

(b)

Fig. 3. The ac signal in unbalanced and balanced detection modes are plotted. The balanced output is almost $6 \mathrm{~dB}$ higher in amplitude than the unbalanced signal. Noise suppression of greater than $17 \mathrm{~dB}$ is achieved.

Fig. 2(a) depicts the experimental setup for balanced detection. A distributed feedback (DFB) laser with 1542-nm wavelength and $0-\mathrm{dBm}$ output power is employed as the optical source. It is amplified by an EDFA and then filtered by an optical bandpass filter with $2-\mathrm{nm}$ bandwidth. The microwave signal was modulated onto the optical carrier by an X-coupled Mach-Zehnder modulator (MZM), which produces two complementary outputs for the balanced VMDP. The outputs are coupled to the balanced VMDP by two lensed fibers. To maximize the signal enhancement and noise cancellation, it is important to match the amplitudes and phases of the two detected microwave signals. In our experiment, a variable attenuator was used to match the amplitudes of the photocurrents. Typical balance between the VMDP was within $2 \%$ of the total photocurrent reading, which was limited to 4 $\mathrm{mA}$ in our current experiments. Since the accuracy of cutting optical fiber lengths is about $1 \mathrm{~mm}$, a variable delay line was used to ensure equal optical path lengths between the two fibers, which is necessary to produce the broad-band $180^{\circ}$ out-of-phase RF signals for balanced VMDP. The output from the balanced VMDP was measured using a spectrum analyzer. A common mode rejection ratio of $-27 \mathrm{~dB}$ has been achieved.

Balanced detection is achieved by applying a bias of $8 \mathrm{~V}$ between the two ground electrodes of the CPW. A custommade high-frequency probe with an integrated dc-blocking capacitor on one ground probe is used to collect the microwave output signal. The dc difference photocurrent is monitored through the bias-T connected to the probe. To verify the balanced detection, we modulated the optical input at $8 \mathrm{GHz}$ and varied the delay of the fiber-optic delay line from 0 to 50 mm. Fig. 2(b) shows the RF signal versus the delay. When the
RF signals have $0^{\circ}$ phase difference, the ac output is cancelled in balanced mode. At $180^{\circ}$ phase difference, the RF signal is amplified, the extinction ratio is more than $44 \mathrm{~dB}$.

Fig. 3 shows the RF spectrum of the output from the balanced VMDP in the unbalanced (only one waveguide is illuminated) and the balanced mode. Suppression of the noise floor by $17 \mathrm{~dB}$ has been observed in the balanced mode over a wide frequency range from 6 to $15 \mathrm{GHz}$. The signal is also enhanced by $6 \mathrm{~dB}$.

\section{CONCLUSION}

We have successfully designed, fabricated, and experimentally demonstrated a balanced velocity-matched distributed photodetector (VMDP) with both impedance and velocity matching. The device exhibits a very low dark current and a high external quantum efficiency of $0.60 \mathrm{~A} / \mathrm{W}$. Using the balanced VMDP, the RIN and EDFA-added noise have been suppressed by $17 \mathrm{~dB}$, and the RF signal has been enhanced by $6 \mathrm{~dB}$. This was the first integration of balanced detectors with high-power, high-speed distributed photodetectors for high performance RF photonic links.

\section{ACKNOWLEDGMENT}

The authors would like to thank Dr. D. T. K. Tong of Lucent Technologies-Bell Laboratories for his helpful suggestion about the experimental setup and Dr. X. J. Meng of UCLA for his helpful discussion about the measurements.

\section{REFERENCES}

[1] L. T. Nichols, K. J. Williams, and R. D. Esman, "Optimizing the ultrawide-band photonic link," IEEE Trans. Microwave Theory Tech., vol. 45, pp. 1384-1389, Aug. 1997.

[2] K. J. Williams and R. D. Esman, "Optically amplified down converting link with shot-noise limited performance," IEEE Photon Technol. Lett., vol. 8, pp. 148-150, Jan. 1996.

[3] D. Trommer, A. Umbach, W. Passenberg, and G. Unterborsch, "A monolithically integrated balanced mixer OEIC on InP for coherent receiver applications," IEEE Photon Technol. Lett., vol. 5, pp. 1038-1040, Sept. 1993.

[4] F. Ghirardi, A. Bruno, B. Mersali, J. Brandon, L. Giraudet, A. Scavennec, and A. Carenco, "Monolithic integration of an InP based polarization diversity heterodyne photoreceiver with electrooptic adjustability," J. Lightwave Technol., vol. 13, pp. 1536-1549, July 1995.

[5] R. J. Deri, E. C. M. Pennings, A. Scherer, A. S. Gozdz, C. Caneau, N. C. Andreadakis, V. Shah, L. Curtis, R. J. Hawkins, J. B. D. Soole, and J.I. Song, "Ultracompact monolithic integration of balanced, polarization diversity photodetectors for coherent lightwave receivers," IEEE Photon Technol. Lett., vol. 4, pp. 1238-1240, Nov. 1992.

[6] L. Y. Lin, M. C. Wu, T. Itoh, T. A. Vang, R. E. Muller, D. L. Sivco, and A. Y. Cho, "Velocity-matched distributed photodetectors with highsaturation power and large bandwidth," IEEE Photon Technol. Lett., vol. 8, pp. 1376-1378, Oct. 1996.

[7] T. Chau, L. Fan, D. T. K. Tong, S. Mathai, M. C. Wu, D. L. Sivco, and A Y. Cho, "Long wavelength velocity-matched distributed photodetectors for RF fiber optic links," Electron. Lett., vol. 34, no. 14, pp. 1422-1424, July 1998.

[8] E. H. Bottcher, H. Pfitzenmaier, E. Droge, and D. Bimberg, "Millimeterwave coplanar waveguide slow wave transmission lines on InP," Electron. Lett., vol. 32, no. 15, pp. 1377-1378, 1996.

[9] E. Sano, M. Yoneama, T. Enoki, and T. Tamamura, "Performance dependance of InGaAs MSM photodetectors on barrier-enhancement layer structures," Electron. Lett., vol. 28, no. 13, pp. 1220-1221, 1992.

[10] W. A. Wohlmuth, P. Fay, and I. Adesida, "Dark current suppression in GaAs metal-semiconductor-metal photodetectors," IEEE Photon Technol. Lett., vol. 8, pp. 1061-1064, Aug. 1996. 